\title{
Glycosphingolipids Prevent APAP and HMG-CoA Reductase Inhibitors-mediated Liver Damage: A Novel Method for "Safer Drug" Formulation that Prevents Drug-induced Liver Injury
}

\author{
Meir Mizrahi, Tomer Adar, Gadi Lalazar, Dean Nachman, Madi El Haj, Ami Ben Ya'acov, \\ Yoav Lichtenstein, Yehudit Shabat, Dimitri Kanovich, Lida Zolotarov and Yaron Ilan*
}

Gastroenterology and Liver Units, Department of Medicine, Hebrew University-Hadassah Medical Center, Jerusalem, Israel

\begin{abstract}
Background and Aims: Acetaminophen (APAP) and HMGCoA reductase inhibitors are common causes of drug-induced liver injury (DILI). This study aimed to determine the ability to reduce APAP- and statins-mediated liver injury by using formulations that combine glycosphingolipids and vitamin E. Methods: Mice were injected with APAP or with statins and treated before and after with $\beta$-glucosylceramide (GC), with or without vitamin $\mathrm{E}$. Mice were followed for changes in liver enzymes, liver histology, hepatic expression of JNK, STAT3 and caspase 3, as well as intrahepatic natural killer T cells (NKT) and the serum cytokine levels by flow cytometry. Results: Administration of GC before or after APAP alleviated the liver damage, as noted by a reduction of the liver enzymes, improvement in the liver histology and decreased hepatic caspase 3 expression. Beneficial effect was associated with a reduction of the intrahepatic NKT, JNK expression in the liver, and increased glutathione in the liver, and decreased TNF- $\alpha$ serum levels. Synergistic effect of co-administration of GC with vitamin E was observed. Similar protective effect of GC on statin-mediated liver damage was documented by a reduction in liver enzymes and improved liver histology, which was mediated by reduction of NKT, increased STAT3 expression in the liver, and reduced the TGF- $\beta$ and IL17 levels. Conclusions: $\beta$-glycosphingolipids exert a hepatoprotective effect on APAP- and statins-mediated liver damage. Vitamin E exerted a synergistic effect to that of GC. The generation of "safer drug" formulations, which include an active molecule combined with a hepatoprotective adjuvant, may provide an answer to the real unmet need of DILI.

Citation of this article: Mizrahi M, Adar T, Lalazar G, Nachman $D$, Haj ME, Ya'acov AB, et al. Glycosphingolipids prevent APAP and HMG-CoA reductase inhibitors-mediated liver damage: A novel method for "safer drug" formulation that prevents drug-induced liver injury. J Clin Transl Hepatol 2018;6(2): 127-134. doi: 10.14218/JCTH.2017.00071.
\end{abstract}

Keywords: Acetaminophen; Statins; Glycosphingolipids.

Abbreviations: ALT, alanine aminotransferase; APAP, acetaminophen; AST, aspartate aminotransferase; DILI, drug-induced liver injury; GC, $\beta$-glucosylceramide; IFN- $\gamma$, interferon-gamma; IL, interleukin; IP, intraperitoneally; NAC, $\mathrm{N}$-acetylcysteine; NAPQI, N-acetyl-p-benzoquinone imine; NKT, natural killer T cell; PBS, phosphatebuffered saline; PO, per os; TGF- $\beta$, transforming growth factor-beta; TNF- $\alpha$, tumor necrosis-alpha.

Received: 4 November 2017; Revised: 8 December 2017; Accepted: 23 January 2018

*Correspondence to: Yaron Ilan, Department of Medicine, Hebrew UniversityHadassah Medical Center, P.O.B 12000, Jerusalem, IL-91120, Israel. Tel: +972-26777816, Fax: +972-2-6431021, E-mail: ilan@hadassah.org.il
Introduction

Idiosyncratic drug-induced liver injury (DILI) is a major health problem. It is an important cause of morbidity and mortality following the drugs taken in therapeutic doses. Hepatotoxicity is a major obstacle in drug development and may lead to withdrawal or restricted use, even after approval. ${ }^{1}$ Its incidence is estimated at 19 cases per 100,000 per year. $^{2}$ Moreover, DILI accounts for $2-5 \%$ of patients requiring hospitalization for jaundice and $10 \%$ of cases of hepatitis in all adults.

DILI ranges from an asymptomatic elevation in transaminases to severe hepatitis and acute liver failure. ${ }^{3}$ The pathogenesis of DILI is due to a combination of host, drug metabolite and environmental factors. ${ }^{1}$ A genome-wide association study suggested an association between the human leukocyte antigen class II and susceptibility to DILI. ${ }^{4}$ The treatment for DILI is largely supportive. Hepatic inflammation is a common feature of DILI and is attributed to the innate immune response followed by an effect on adaptive immunity, resulting in liver damage. ${ }^{5,6}$ The Thelper cell-mediated immune responses were implicated in the pathogenesis.

Acetaminophen (APAP) is a commonly used antipyretic and analgesic agent. APAP overdose and the resulting hepatotoxicity may lead to fatal hepatotoxicity. ${ }^{7}$ APAP accounts for up to $50 \%$ of all adult cases of acute liver failure in the USA. ${ }^{8}$ About two-thirds of the adult overdoses are associated with suicide attempts, but the rest are inadvertent, often due to the long-term chronic use. ${ }^{8,9}$ In pediatric populations, the majority of the APAP overdoses are unintentional. APAP toxicity at the therapeutic doses of $<4 \mathrm{~g} /$ day were reported due to unknown contributing factors.

APAP-mediated liver injury is not directly caused by the drug itself, but by a formation of the toxic metabolite, $\mathrm{N}$-acetyl-p-benzoquinone imine (NAPQI), generated through cytochrome P-450. ${ }^{9}$ Glutathione is an endogenous antioxidant, and its hepatic stores combine with the toxic metabolite to prevent liver cell injury. When glutathione stores are depleted by overproduction of NAPQI, it binds to liver cell proteins thereby inducing hepatic necrosis. ${ }^{9}$ Studies have suggested a role for the host immune response in the pathogenesis of APAP hepatotoxicity. ${ }^{10}$

Cytokine production by the activated Kupffer cells, variation in the expression of the lymphocyte CD44 gene and natural killer T cells (NKT) were all suggested to play a role in the APAP-associated liver injury. ${ }^{11-14} \mathrm{~N}$-acetylcysteine (NAC) is the most effective and only drug to prevent progression to liver failure with APAP hepatotoxicity. ${ }^{15}$ Treatment with NAC 
has been shown to increase glutathione levels. ${ }^{16}$ However, its therapeutic window is narrow, and in order to be effective its administration should take place within several hours of ingestion, a period of time during which many patients are still asymptomatic. ${ }^{9}$

The HMG-CoA reductase inhibitors (statins) are widely prescribed drugs. Up to $3 \%$ of the patients will develop mild elevations in serum aminotransferases; however, clinically overt DILI is rare. ${ }^{17} \mathrm{~A}$ prospective study of 1,188 cases of DILI attributed 22 cases to statins. The latency to onset of liver injury ranged from 34 days to 10 years, with the average peak alanine aminotransferase (ALT) levels being $892 \mathrm{U} / \mathrm{L}$ and of total being bilirubin $6.1 \mathrm{mg} / \mathrm{dL}$. DILI from statins typically presents with an acute hepatocellular liver injury pattern, although mixed or cholestatic injury patterns have also been reported. ${ }^{17,18}$ Cases of severe hepatotoxicity show autoimmune phenotypes, which is mediated by an effect on the immune system and cell membranes. ${ }^{19-24}$

The aim of the present study was to determine the ability to reduce APAP- and HMG-CoA reductase inhibitors-mediated liver injury by using a formulation that combined glycosphingolipids with the active molecule.

\section{Methods}

\section{Animals}

Male C57BI/6 mice (12-weeks-old) were obtained from the Harlan Laboratories (Jerusalem, Israel) and maintained in the Animal Core of the Hadassah-Hebrew University Medical School. To determine the mechanism of action and dependency on NKT, $\mathrm{CD} 1 \mathrm{~d}^{-/-}$mice from a $\mathrm{C} 57 \mathrm{BI} / 6$ background were kindly provided by Dr. L. Van Kaer (Vanderbilt University, Nashville, TN, USA). The J- $\alpha-18$ KO mice were kindly provided by Dr. M. Taniguchi (Riken Research Center for Allergy and Immunology, Yokohama, Japan). All mice were used at 11-12 weeks of age and were maintained in the Animal Core of the Hadassah-Hebrew University Medical School. All the mice were administered standard laboratory chow and water ad libitum and kept in a 12-h light/dark cycle. Animal experiments were carried out according to the guidelines of the Hebrew University-Hadassah Institutional Committee for Care and Use of Laboratory Animals, and with the committee's approval. Experiments were repeated twice for parts B and C (see below in the Experimental groups subsection).

\section{Preparation of glycolipids}

$\beta$-glucosylceramide (GC) was purchased from Avanti Polar Lipids (Alabaster, AL, USA). The GC was dissolved in a mixture of $30 \%$ Cremophor (Sigma, Rehovot, Israel) and ethanol (1:1) in phosphate-buffered saline (PBS). GC was orally administered at a dose of $1 \mathrm{mg} / \mathrm{kg}$.

\section{Preparation of APAP}

APAP (Tiptipot liquid; CTS Group, Tel-Aviv, Israel) was administered intraperitoneally in a dose of $500 \mathrm{mg} / \mathrm{kg}$ dissolved in Cremophor.

\section{Preparation of statins}

Simvastatin was dissolved in water and orally administered at $5 \mathrm{mg} / \mathrm{kg}$ daily.

\section{Experimental groups}

Part A: to assess the protective effect of GC on APAPmediated liver damage. Five groups of mice, 15 per group, were studied. The mice in all the groups were fasted for $8 \mathrm{~h}$ before APAP administration. The mice were treated with GC [100 mcg, per os (PO)] $2 \mathrm{~h}$ before (Group B) or $2 \mathrm{~h}$ after (Group C) APAP administration. The mice in the control group (Group A) were treated with PBS via oral gavage. CD1d ${ }^{-/-}$mice (Group D) and J- $\alpha-18$ deficient mice (Group E) were treated with PBS via oral gavage.

Part B: to assess the protective effect of GC and vitamin E on APAP-mediated liver damage. To determine the synergistic effect of GC and vitamin E in APAP-induced liver injury, four groups of mice injected with APAP intraperitoneally (IP) were treated with vitamin $\mathrm{E}$ alone $(125 \mathrm{mg} /$ dose, $\alpha$-Tocopherol; Cat. No. 258024; Sigma) or GC alone (PO), or with a combination of GC with vitamin E (PO). Treatments were administered prior to APAP. The control group received APAP alone.

Part C: to assess the protective effect of GC on statinmediated liver damage. C57BI/6 mice were treated with simvastatin ( $1.25 \mathrm{mg} /$ day) for 8 weeks via oral gavage. The mice were divided into three groups treated concomitantly with PBS via oral gavage (Group A), $100 \mathrm{mcg} /$ dose of GC IP (Group B), or via oral gavage (Group C).

\section{Assessment of the effect of $\beta$-glycosphingolipids on liver injury}

Serum was collected and assayed for serum ALT and aspartate aminotransferase (AST) $24 \mathrm{~h}$ after the APAP administration. Pathological sections were prepared from all the mice in all the groups at the end of the study, and were stained with hematoxylin and eosin. Caspase 3 staining was performed on the liver sections to determine the antiapoptotic effect of the treatment using anti-caspase 3 antibodies (Cat. No. c8487; Sigma).

\section{Assessment of the effect of $\beta$-glycosphingolipids on the immune response}

Isolation of splenocytes and hepatic lymphocytes: Splenocytes and hepatic lymphocytes were isolated as previously described. ${ }^{25,26}$ Approximately $1 \times 10^{6}$ cells/mouse liver were recovered.

Flow cytometry for lymphocyte subsets: Flow cytometry was performed following the lymphocyte isolation using $1 \times 10^{6}$ lymphocytes in $100 \mu \mathrm{L}$ PBS. To determine the percentage of NKT lymphocytes, PE-Cy5 anti-mouse CD3 and PE antimouse NK1.1 PE-conjugated CD25-specific (PC61) antibodies were used (eBioscience, San Diego, CA, USA). CD4 and CD8 subsets were detected using PE-Cy5 anti-mouse CD3 and PEanti-mouse CD4 or CD8 antibodies. The data were analyzed using either the Consort 30 two-color contour plot program (Becton Dickinson, Oxnard, CA, USA) or the CELLQuest 25 program.

Determination of cellular glutathione activity: Cell lysates were prepared as follows: $2 \times 10^{6}$ cells were collected by centrifugation at $1,000 \mathrm{~g}$ for $10 \mathrm{~m}$ at $4^{\circ} \mathrm{C}$. The cells were lysed by homogenization in 1-2 $\mathrm{mL}$ of cold buffer containing $50 \mathrm{mM}$ MES ( $\mathrm{pH} \mathrm{6-7)}$ and $1 \mathrm{mM}$ EDTA. Centrifugation at $10,000 \mathrm{~g}$ for $15 \mathrm{~m}$ at $4^{\circ} \mathrm{C}$ was performed and the supernatant was assayed for glutathione using a commercially available kit (QuantiChrom $^{\mathrm{TM}}$ Glutathione Assay Kit, DIGT-250; Bioassay Systems, Hayward, CA, USA). 
Phosphorylation of STAT3 and JNK: Splenocytes were lysed in RIPA buffer containing 50 mM Tris, pH 7.4, 1\% NP-40, $150 \mathrm{mM} \mathrm{NaCl}, 0.1 \%$ sodium dodecyl sulfate, $0.5 \%$ sodium deoxycholate and protease inhibitors cocktail $(5 \mu \mathrm{L} / \mathrm{mL}$; Sigma). Lysates were centrifuged and protein concentration was determined by Bradford assay (Bio-Rad, Hercules, CA, USA). Proteins were detected with phospho-specific STAT3 and JNK (Cell Signaling, Danvers, MA, USA). $\beta$-actin (Abcam, Cambridge, UK) was used as loading control. All blots were developed with horseradish peroxidase-conjugated secondary antibodies (Jackson Laboratory, Bar Harbor, ME, USA) and Western Blot-Luminol Reagent (Santa Cruz Biotechnology, Dallas, TX, USA).

Serum cytokines: The serum levels of tumor necrosis factor-alpha (TNF- $\alpha$ ), tumor growth factor-beta (TGF- $\beta$ ), and interleukin (IL) 17 were tested in all the groups using a commercially available enzyme-linked immunoassay kit (Genzyme, Cambridge, MA, USA).

\section{Statistical analysis}

The comparison of two independent groups' means was performed using a two-sided student's $t$ test. A $p$ value smaller than 0.05 was considered statistically significant.

\section{Results}

\section{Protective effect of glycosphingolipids on APAP- mediated liver injury}

Fig. 1 shows the protective effect of GC on APAP-mediated damage. Panel A shows a significant decrease in the ALT serum levels noted in the mice treated before (Group B, 5222 IU/L) or after (Group C, 5034 IU/L) APAP administration, as compared to the untreated controls (Group A, 12150 $\mathrm{IU} / \mathrm{L}, p<0.05 ; n=7$ ). Panel $\mathrm{B}$ shows a marked improvement in the histological damage with a decrease in the cell necrosis of the GC-treated mice. A marked decrease in caspase 3 is shown in the GC-treated groups, which may support a decrease in cell apoptosis.

Fig. 2 shows a significant decrease in the intrahepatic NKT (Panel A). Interestingly, no damage was noted in CD1d ${ }^{-/-}$or in the J- $\alpha-18 \mathrm{KO}$ mice (data not shown), further supporting a role for the NKT in mediating the damage. Panel B shows a decrease in JNK phosphorylation in the mice in Group B, with a moderate decrease in the mice in Group $C$. The beneficial hepatoprotective effect of GC was associated with a significant increase in the liver glutathione levels as shown in Panel $C$, and with a decrease in the TNF- $\alpha$ serum levels noted in the mice in Group C.

\section{Synergistic effect of vitamin E and GC in APAP- mediated liver damage}

Fig. 3 shows a synergistic effect between GC and vitamin E in liver protection. While GC exerted some liver protection, the combination of GC with vitamin E exerted a marked alleviation of the APAP-mediated liver damage when administered prior to induction of damage, as seen in Panel A $(p<0.05)$. The histological sections show a marked improvement of liver damage in the GC with vitamin E-treated mice, as compared to the mice treated with GC alone. Vitamin E had only minimal effect on histology (data not shown).

\section{Protective effect of glycosphingolipids on statin- mediated liver injury}

Fig. 4 shows the beneficial effect of both the oral and IP administration of GC. Panel A shows a significant decrease in the ALT and AST serum levels of the GC-treated mice $(n=7)$. Panel B shows a marked decrease in the cell necrosis in the histological sections from GC-treated mice.

\section{A}

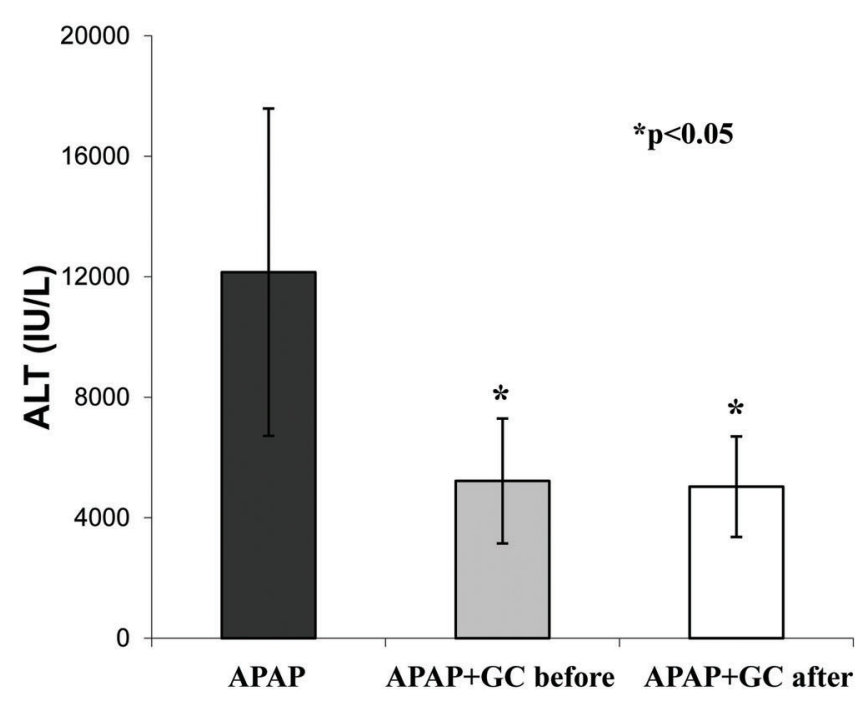

B

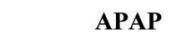

APAP

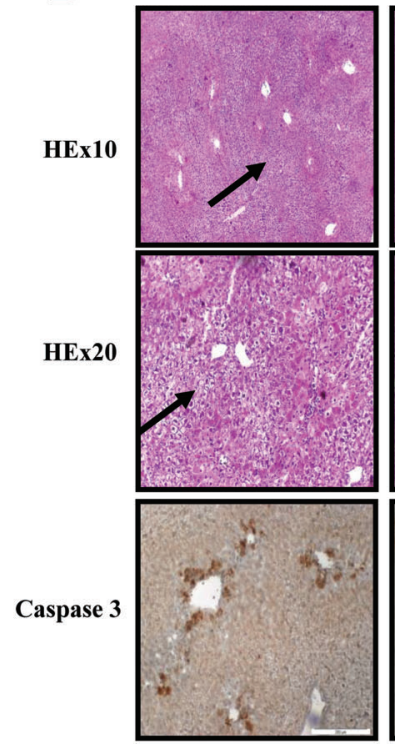

APAP + GC before

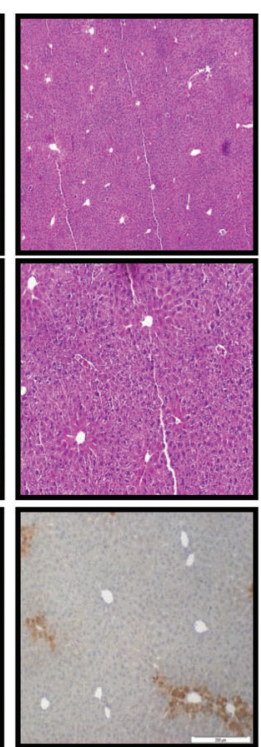

APAP + GC after

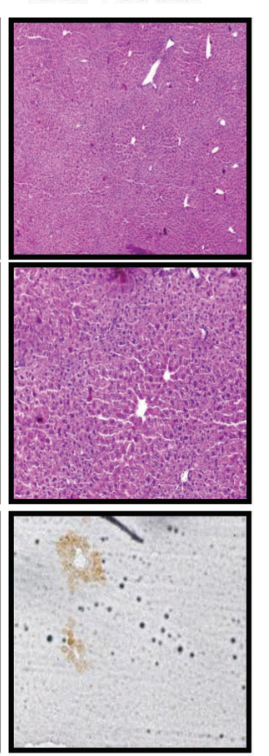

Fig. 1. Protective effect of $\boldsymbol{\beta}$-glucosylceramide from APAP-mediated liver damage. The mice were treated with GC before (Group $B$ ) or after (Group $C$ ) injection of APAP, and compared to the controls (Group A) $(* p<0.05)$. (A) Mice were followed to determine changes in ALT serum levels. (B) Representative liver sections from the mice in all the groups stained with hematoxylin and eosin, and shown at $10 \times$ and $20 \times$ magnifications. Arrows mark the areas of APAP-associated liver damage. The liver sections were stained for caspase 3 to assess the effect of the treatment on liver apoptosis. 


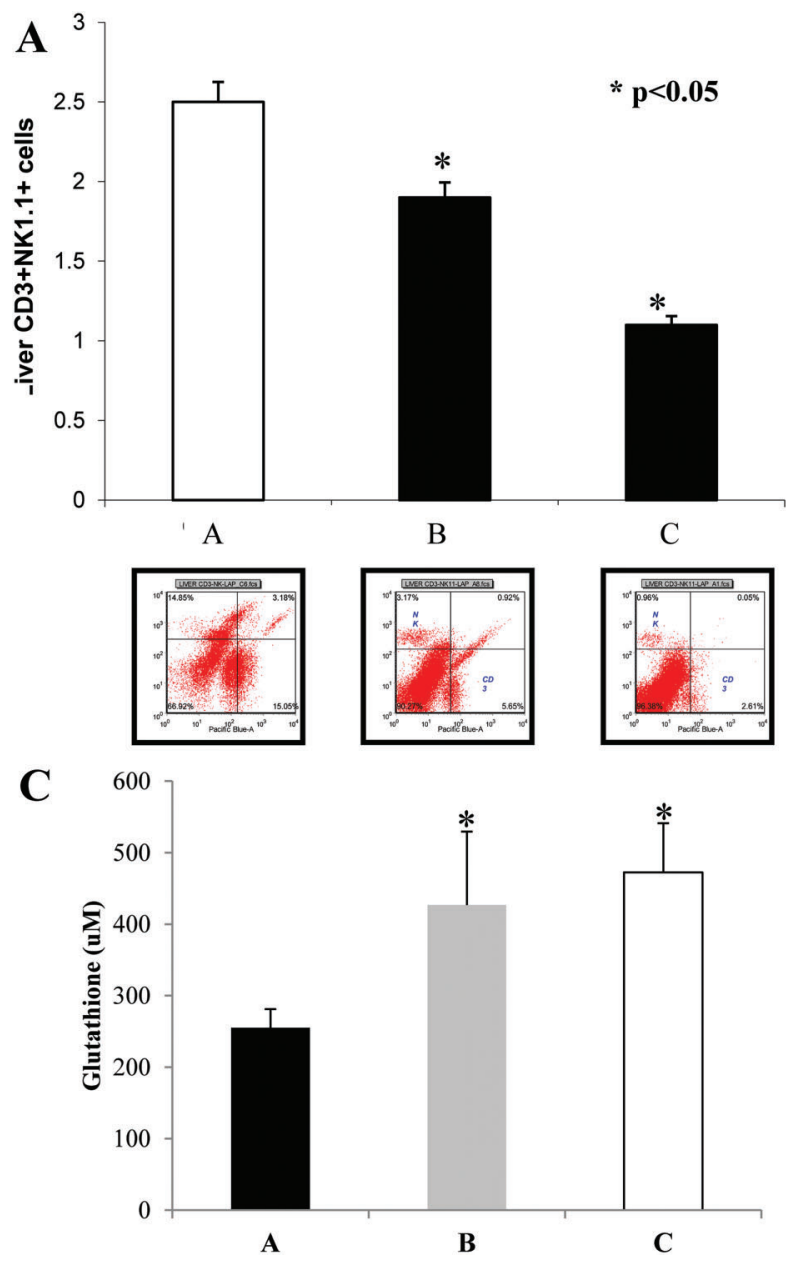

B
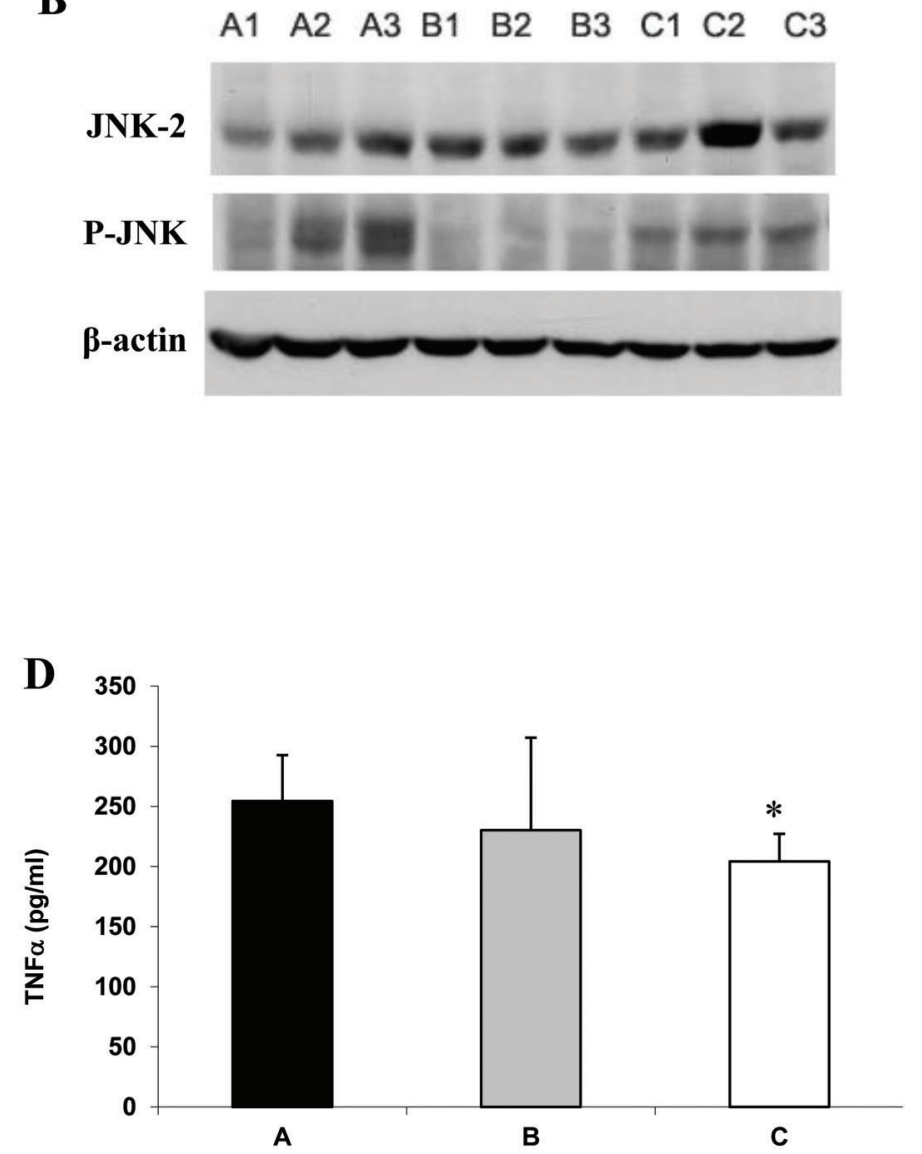

Fig. 2. Effects on NKT, JNK expression, glutathione and TNF- $\alpha$. (A) FACS analysis was conducted for intrahepatic the NKT in all mice in all groups. (B) Western blot analysis of the JNK expression on the liver sections from three mice from each group. (C) Assessment of the effect of the treatment on the glutathione levels in the liver. (D) Assessment of the effect of the treatment on the TNF- $\alpha$ serum levels. ${ }^{*} p<0.05$.

Fig. 5 shows that the beneficial effect of GC was associated with a decrease in intrahepatic NKT and splenic $\mathrm{CD}^{+} \mathrm{CD}^{+}$ subsets of lymphocytes (Panel A). Panel B shows a significant decrease in the IL17 serum levels, along with a significant increase in the TGF- $\beta$ serum levels in the treated mice $(p<0.01)$. Panel $C$ shows an increase in STAT3 phosphorylation in livers of orally treated mice, but a decrease in the IP-treated ones.

\section{Discussion}

The results of the present study support the ability to alleviate APAP- and statins-mediated liver injury by the administration of glycosphingolipids. Glycosphingolipids were previously shown to exert a beneficial effect in several immune-mediated liver disorders. ${ }^{19-22,24,27-34}$ The preliminary data suggest their potential beneficial effects on humans. ${ }^{35}$ An NKT- and an immune environment-dependent mechanism may lay at the basis of their immunoprotective effects. ${ }^{22-24,33,36}$

In the present study, we showed a significant improvement in the hepatocellular injury and an improvement in the histological damage in the mice treated with GC before or after APAP administration. The hepatoprotective effect may be mediated via a decrease in liver apoptosis demonstrated by a decrease in caspase 3 staining in the liver, along with a significant increase in the liver glutathione levels, and a decrease in the TNF- $\alpha$ serum levels.

The present data show that a significant decrease in the intrahepatic NKT occurred in the GC-treated mice, and a lack of APAP-mediated damage in the CD1d ${ }^{-/-}$or in the $\mathrm{J}-\alpha-18$ mice, further supporting a role for NKT in APAP-mediated damage. While their role in liver damage is still being debated, studies have shown that both the natural killer cells and NKT play a role in the APAP-induced liver injury by producing interferongamma (IFN- $\gamma$ ) and modulating chemokine production and recruitment of neutrophils into the liver. ${ }^{37,38}$ Depletion of both the natural killer and NKT cells by anti-NK1.1 significantly protected mice from APAP-induced liver injury. ${ }^{39}$ Part of these effects was attributed to the solvent used. ${ }^{14}$ Recent studies have shown an elevated ketone body production in the NKTdeficient mice, which results in an increased CYP2E1-mediated APAP biotransformation and susceptibility to APAP-mediated damage. ${ }^{13}$

A decrease in JNK phosphorylation was noted in the GCtreated mice. APAP induces JNK signaling and activates the caspase-9-3-dependent cell death pathway. ${ }^{40} \mathrm{JNK}$ activation promotes cell death and APAP-liver injury. ${ }^{41}$ The amelioration of 

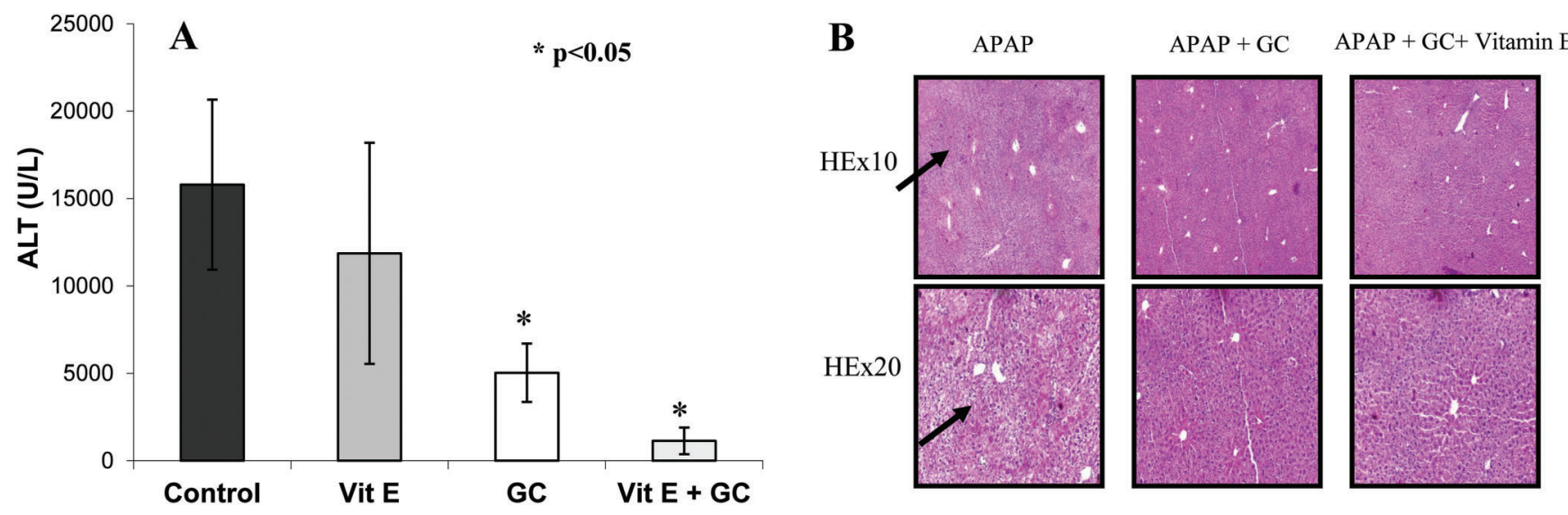

Fig. 3. Synergistic effect of $\boldsymbol{\beta}$-glucosylceramide with vitamin E on alleviation of the APAP-mediated liver damage. (A) Mice were followed to determine the changes in the ALT serum levels. (B) Representative liver sections from mice in all the groups stained with hematoxylin and eosin, and shown at $10 \times$ and $20 \times$ magnifications. $* p<0.05$.

APAP hepatotoxicity is associated with inhibiting the JNK signaling pathway, and it is associated with reducing hepatocyte necrosis, blocking generation of reactive oxygen species, inhibiting oxidative stress, and reducing apoptosis. ${ }^{42}$

A synergistic effect between GC and vitamin E was noted for the APAP-mediated liver protection. This combination led to a marked alleviation of the APAP-mediated liver damage, as noted by a decrease in the liver enzymes and by a marked histological improvement.

Alpha-tocopherols elicit hepatoprotective effects against DILI through the activation of an antioxidant response. ${ }^{43}$ Vitamin E administration prior to APAP administration exerts

A

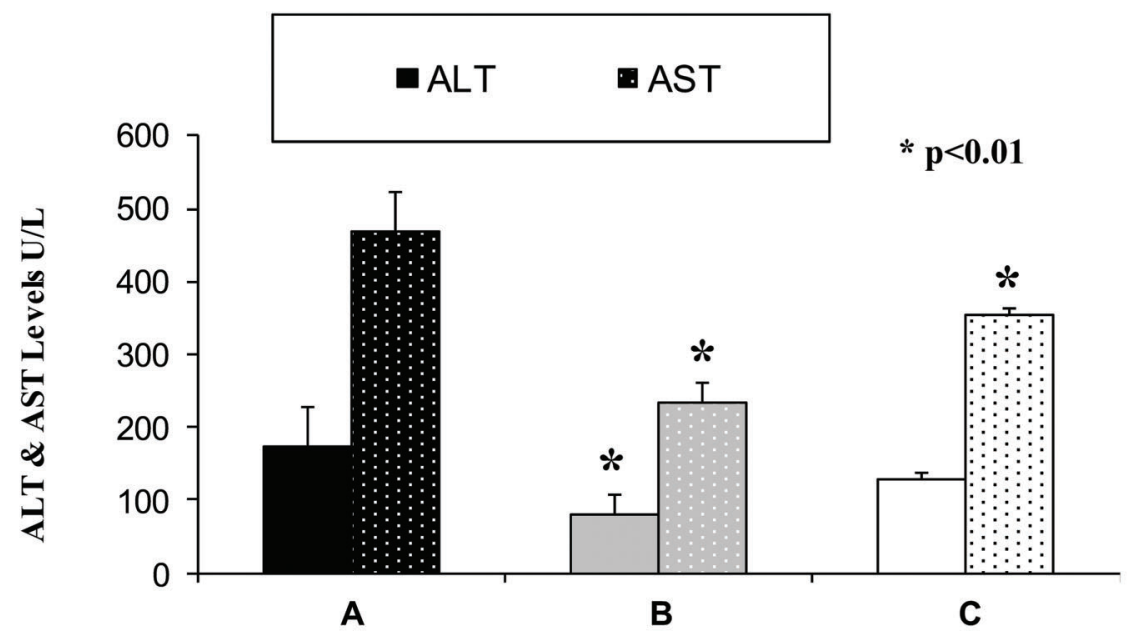

\section{B $\operatorname{HEx} 20$}
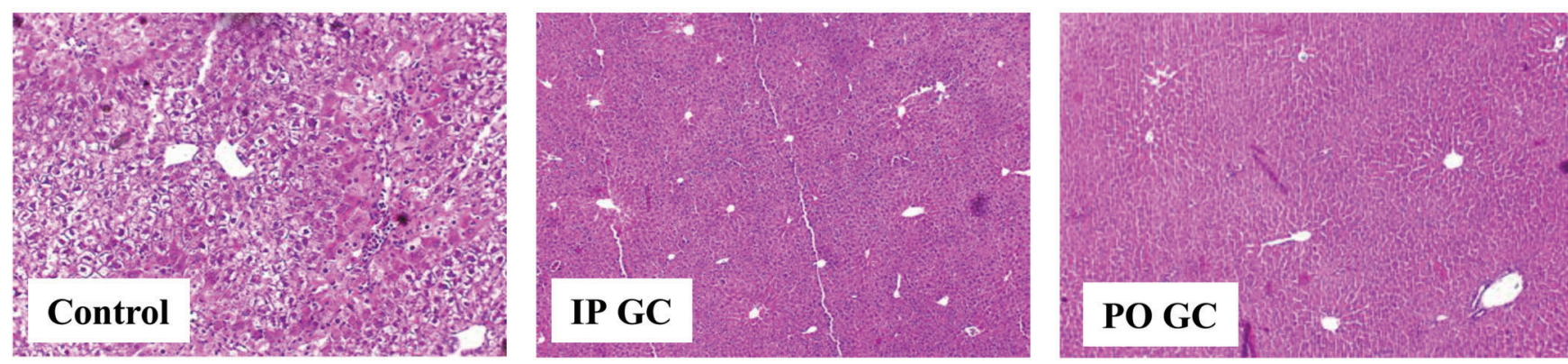

Fig. 4. Protective effect of $\boldsymbol{\beta}$-glucosylceramide from statin-mediated liver damage. Mice were treated with IP GC (Group B) or oral GC (Group C) after injection of APAP and compared to the controls (Group A). (A) Mice were followed for the effect of the treatment on the ALT and AST serum levels. (B) Representative liver sections from mice in all the groups stained with hematoxylin and eosin ( $20 \times$ for control, $10 \times$ for treated). $* p<0.01$. 

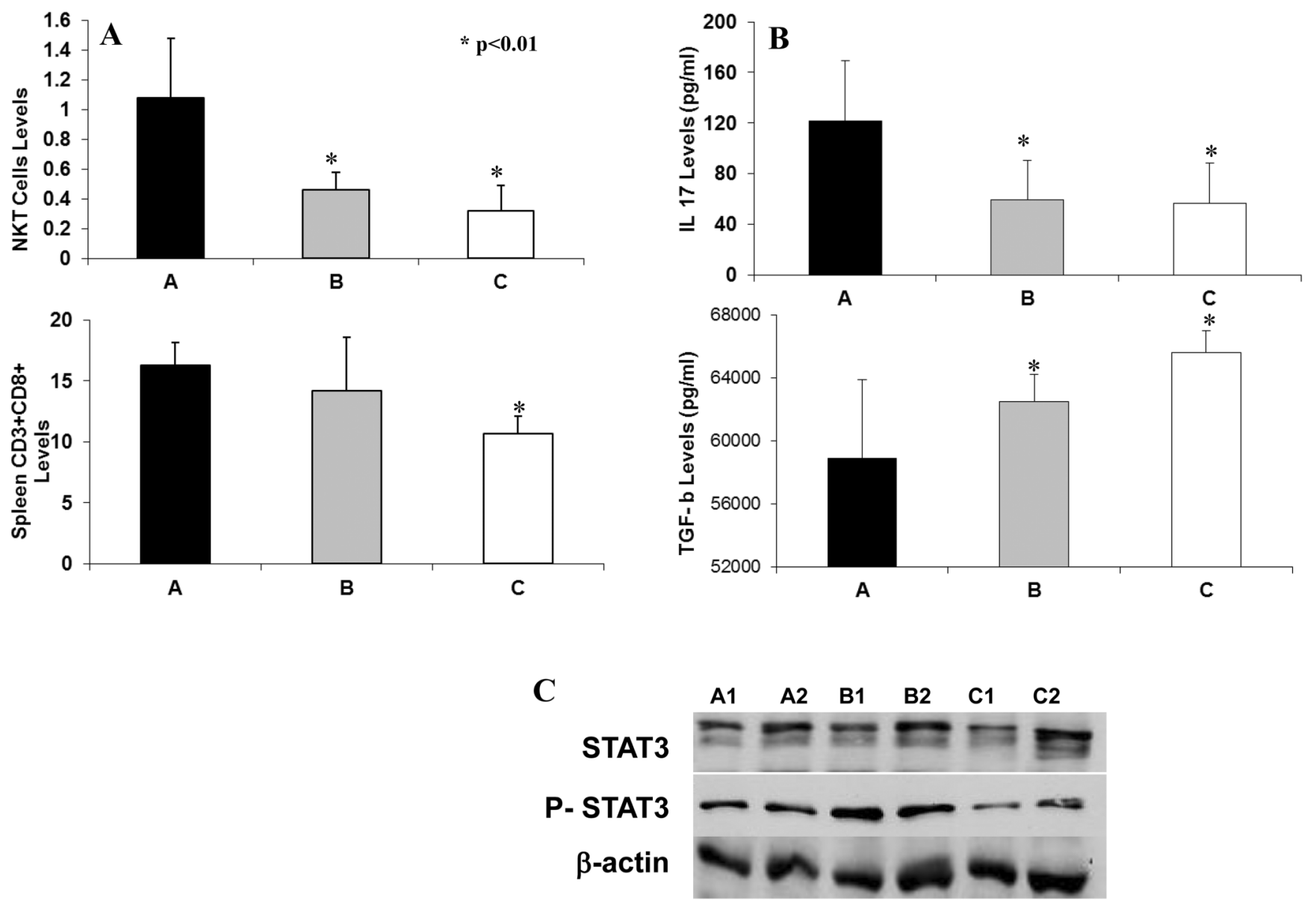

Fig. 5. Effect of therapy on subsets of T lymphocytes. (A) FACS analysis was conducted for the intrahepatic NKT and for splenic CD $3^{+} \mathrm{CD} 8^{+}$in all mice in all the groups. (B) Assessment of the effect of treatment on the TGF- $\beta$ and IL17 serum levels. (C) Western blot for STAT3 expression in the liver, conducted on two mice from each group. ${ }^{*} p<0.01$.

a hepatoprotective effect. ${ }^{44}$ In our study, vitamin $E$ exerted only a mild protective effect. This is in line with the previous data showing that the use of antioxidants such as alpha lipoic acid does not protect the liver fully from APAP-induced acute toxicity. ${ }^{45}$

A beneficial effect of glycosphingolipids was also shown for the statin-mediated liver injury. Both the oral and IP administration of GC was associated with a significant decrease in the ALT and AST serum levels and with a marked decrease in cell necrosis of the liver. The noted beneficial effects in the present study were associated with a decrease in intrahepatic NKT and splenic $\mathrm{CD} 3^{+} \mathrm{CD} 8^{+}$subsets of lymphocytes, a decrease in IL17, and an increase in the TGF- $\beta$ serum levels. Statins have antiinflammatory effects. ${ }^{46}$ The dendritic cells treated with atorvastatin are induced into tolerogenic dendritic cells, increasing the NKT, inhibiting the $\mathrm{CD} 4^{+}$T cell proliferation, and decreasing the levels of IFN- $\gamma$, TNF- $\alpha$ and IL17. ${ }^{47}$ Whether the statinmediated NKT increase is associated with the statin-mediated live damage remains to be shown.

The GC-mediated liver protection was associated with an increase in STAT3 phosphorylation. Statins reduce this IL6induced phosphorylation of STAT3 in hepatocytes. ${ }^{48}$ The STAT3 pathway is protective in murine models of APAP intoxication, and the administration of recombinant STAT3-activating cytokines was suggested as a therapeutic approach. ${ }^{49}$ The protective effect of IL22 from APAP-mediated hepatotoxicity was dependent on STAT3. ${ }^{50}$

In summary, $\beta$-glycosphingolipids exert a hepatoprotective effect on APAP- and statins-mediated liver damage. Vitamin E was shown to exert a synergistic effect to that of GC. The generation of "safer drug" formulations, which include the active molecule with a hepatoprotective adjuvant, may provide an answer to the real unmet need of DILI.

\section{Acknowledgments}

This work was supported in part by a grant from the RoamanEpstein Liver Research Foundation (to YI).

\section{Conflict of interest}

The authors have no conflict of interests related to this publication.

\section{Author contributions}

Conducted the experiments (MM, TA, GL, DN, MEH, ABY, YL, YS, DK LZ), designed the studies and review the data and prepared the manuscript (MM, TA, GL, ABY, YI). 


\section{References}

[1] Devarbhavi H. An update on drug-induced liver injury. J Clin Exp Hepato 2012;2:247-259. doi: 10.1016/j.jceh.2012.05.002.

[2] Björnsson ES. Epidemiology and risk factors for idiosyncratic drug-induced liver injury. Semin Liver Dis 2014;34:115-122. doi: 10.1055/s-0034-1375953.

[3] Andrade RJ, Lucena MI, Kaplowitz N, García-Munoz B, Borraz Y, Pachkoria K, et al. Outcome of acute idiosyncratic drug-induced liver injury: Long-term follow-up in a hepatotoxicity registry. Hepatology 2006;44:1581-1588. doi: 10.1002/hep.21424

[4] Grant LM, Rockey DC. Drug-induced liver injury. Curr Opin Gastroenterol 2012;28:198-202. doi: 10.1097/MOG.0b013e3283528b5d.

[5] Wang $X$, Zhang $L$, Jiang $Z$. T-helper cell-mediated factors in drug-induced liver injury. J Appl Toxicol 2015;35:695-700. doi: 10.1002/jat.3115.

[6] Ju C, Reilly T. Role of immune reactions in drug-induced liver injury (DILI). Drug Metab Rev 2012;44:107-115. doi: 10.3109/03602532.2011.645579.

[7] Larson AM. Acetaminophen hepatotoxicity. Clin Liver Dis 2007;11:525-548. doi: 10.1016/j.cld.2007.06.006.

[8] Amar PJ, Schiff ER. Acetaminophen safety and hepatotoxicity-where do we go from here? Expert Opin Drug Saf 2007;6:341-355. doi: 10.1517/14740338. 6.4.341.

[9] McClain CJ, Price S, Barve S, Devalarja R, Shedlofsky S. Acetaminophen hepatotoxicity: An update. Curr Gastroenterol Rep 1999;1:42-49.

[10] Tujios S, Fontana RJ. Mechanisms of drug-induced liver injury: from bedside to bench. Nat Rev Gastroenterol Hepatol 2011;8:202-211. doi: 10.1038/nrgastro. 2011.22.

[11] Saiman $Y$, Friedman SL. The role of chemokines in acute liver injury. Front Physiol 2012;3:213. doi: 10.3389/fphys.2012.00213.

[12] Fisher JE, McKenzie TJ, Lillegard JB, Yu Y, Juskewitch JE, Nedredal GI, et al. Role of Kupffer cells and toll-like receptor 4 in acetaminophen-induced acute liver failure. J Surg Res 2013;180:147-155. doi: 10.1016/j.jss. 2012.11.051.

[13] Martin-Murphy BV, Kominsky DJ, Orlicky DJ, Donohue TM Jr, Ju C. Increased susceptibility of natural killer T-cell-deficient mice to acetaminophen-induced liver injury. Hepatology 2013;57:1575-1584. doi: 10.1002/hep.26134.

[14] Masson MJ, Carpenter LD, Graf ML, PohI LR. Pathogenic role of natural killer T and natural killer cells in acetaminophen-induced liver injury in mice is dependent on the presence of dimethyl sulfoxide. Hepatology 2008;48: 889-897. doi: 10.1002/hep.22400.

[15] Chun LJ, Tong MJ, Busuttil RW, Hiatt JR. Acetaminophen hepatotoxicity and acute liver failure. J Clin Gastroenterol 2009;43:342-349. doi: 10. 1097/MCG.0b013e31818a3854.

[16] Dodd S, Dean O, Copolov DL, Malhi GS, Berk M. N-acetylcysteine for antioxidant therapy: pharmacology and clinical utility. Expert Opin Biol Ther 2008;8:1955-1962. doi: 10.1517/14728220802517901.

[17] Russo MW, Hoofnagle JH, Gu J, Fontana RJ, Barnhart H, Kleiner DE, et al. Spectrum of statin hepatotoxicity: experience of the drug-induced liver injury network. Hepatology 2014;60:679-686. doi: 10.1002/hep.27157.

[18] Russo MW, Scobey M, Bonkovsky HL. Drug-induced liver injury associated with statins. Semin Liver Dis 2009;29:412-422. doi: 10.1055/s-00291240010.

[19] Lalazar G, Ben Ya'acov A, Eliakim-Raz N, Livovsky DM, Pappo O, Preston S, et al. Beta-glycosphingolipids-mediated lipid raft alteration is associated with redistribution of NKT cells and increased intrahepatic CD8+ T lymphocyte trapping. J Lipid Res 2008;49:1884-1893. doi: 10.1194/jlr.M800113JLR200.

[20] Ben Ya'acov A, Lalazar G, Livovsky DM, Kanovich D, Axelrod E, Preston S, et al. Decreased STAT-1 phosphorylation by a thio analogue of beta-Dglucosylceramide is associated with altered NKT lymphocyte polarization. Mol Immunol 2009;47:526-533. doi: 10.1016/j.molimm.2009.07.030.

[21] Lalazar G, Ben Ya'acov A, Lador A, Livovsky DM, Pappo O, Preston S, et al. Modulation of intracellular machinery by beta-glycolipids is associated with alteration of NKT lipid rafts and amelioration of concanavalin-induced hepatitis. Mol Immunol 2008;45:3517-3525. doi: 10.1016/j.molimm.2008. 05.009.

[22] El Haj M, Ben Ya'acov A, Lalazar G, Ilan Y. Potential role of NKT regulatory cell ligands for the treatment of immune mediated colitis. World J Gastroenterol 2007;13:5799-5804.

[23] Zigmond E, Preston S, Pappo O, Lalazar G, Margalit M, Shalev Z, et al. Betaglucosylceramide: a novel method for enhancement of natural killer $T$ lymphoycte plasticity in murine models of immune-mediated disorders. Gut 2007;56:82-89. doi: 10.1136/gut.2006.095497.

[24] Ilan Y. Compounds of the sphingomyelin-ceramide-glycosphingolipid pathways as secondary messenger molecules: new targets for novel therapies for fatty liver disease and insulin resistance. Am J Physiol Gastrointest Liver Physiol 2016;310:G1102-G1117. doi: 10.1152/ajpgi.00095.2016.

[25] Trop S, Samsonov D, Gotsman I, Alper R, Diment J, Ilan Y. Liver-associated lymphocytes expressing NK1.1 are essential for oral immune tolerance induction in a murine model. Hepatology 1999;29:746-755. doi: 10 . 1002/hep.510290334

[26] Falcone M, Facciotti F, Ghidoli N, Monti P, Olivieri S, Zaccagnino L, et al. Upregulation of $C D 1 d$ expression restores the immunoregulatory function of NKT cells and prevents autoimmune diabetes in nonobese diabetic mice. J Immunol 2004;172:5908-5916.

[27] Lalazar G, Preston S, Zigmond E, Ben Yáacov A, Ilan Y. Glycolipids as immune modulatory tools. Mini Rev Med Chem 2006;6:1249-1253.

[28] Lalazar G, Ben Ya'acov A, Livovsky DM, El Haj M, Pappo O, Preston S, et al. Beta-glycoglycosphingolipid-induced alterations of the STAT signaling pathways are dependent on CD1d and the lipid raft protein flotillin-2. Am J Pathol 2009;174:1390-1399. doi: 10.2353/ajpath.2009.080841.

[29] Mizrahi M, Lalazar G, Ben Ya'acov A, Livovsky DM, Horowitz Y, Zolotarov L, et al. Beta-glycoglycosphingolipid-induced augmentation of the anti-HBV immune response is associated with altered CD8 and NKT lymphocyte distribution: a novel adjuvant for HBV vaccination. Vaccine 2008;26: 2589-2595. doi: 10.1016/j.vaccine.2008.03.026.

[30] Zigmond E, Shalev Z, Pappo O, Alper R, Zolotarov L, Ilan Y. NKT lymphocyte polarization determined by microenvironment signaling: a role for CD8+ lymphocytes and beta-glycosphingolipids. J Autoimmun 2008;31:188-195. doi: 10.1016/j.jaut.2008.07.003.

[31] Zigmond E, Tayer-Shifman O, Lalazar G, Ben Ya'acov A, Weksler-Zangen S, Shasha $D$, et al. $\beta$-glycosphingolipids ameliorated non-alcoholic steatohepatitis in the Psammomys obesus model. J Inflamm Res 2014;7:151-158. doi: 10.2147/JIR.S50508.

[32] Zigmond E, Zangen SW, Pappo O, Sklair-Levy M, Lalazar G, Zolotaryova L, et al. Beta-glycosphingolipids improve glucose intolerance and hepatic steatosis of the Cohen diabetic rat. Am J Physiol Endocrinol Metab 2009;296: E72-E78. doi: 10.1152/ajpendo.90634.2008.

[33] Ilan Y. Alpha versus beta: are we on the way to resolve the mystery as to which is the endogenous ligand for natural killer T cells? Clin Exp Immunol 2009;158:300-307. doi: 10.1111/j.1365-2249.2009.04030.x.

[34] Livovsky DM, Lalazar G, Ben Ya'acov A, Pappo O, Preston S, Zolotaryova L, et al. Administration of beta-glycolipids overcomes an unfavorable nutritional dependent host milieu: a role for a soy-free diet and natural ligands in intrahepatic CD8+ lymphocyte trapping and NKT cell redistribution. Int Immunopharmacol 2008;8:1298-1305. doi: 10.1016/j.intimp.2008.05. 005.

[35] Zigmond E, Lalazar G, Pappo O, Zangen SW, Levy Sklair M, Hemed N, et al. Treatment of non-alcoholic steatohepatitis by B-glucosylceramide: A phase I/II clinical study. Hepatology 2006;44:180A. doi: 10.1002/hep. 21394.

[36] Margalit M, Abu Gazala S, Alper R, Elinav E, Klein A, Doviner V, et al. Glucocerebroside treatment ameliorates ConA hepatitis by inhibition of NKT lymphocytes. Am J Physiol Gastrointest Liver Physiol 2005;289:G917-G925. doi: 10.1152/ajpgi.00105.2005.

[37] Liu ZX, Han D, Gunawan B, Kaplowitz N. Neutrophil depletion protects against murine acetaminophen hepatotoxicity. Hepatology 2006;43: 1220-1230. doi: 10.1002/hep.21175.

[38] Krenkel O, Mossanen JC, Tacke F. Immune mechanisms in acetaminopheninduced acute liver failure. Hepatobiliary Surg Nutr 2014;3:331-343. doi: 10.3978/j.issn.2304-3881.2014.11.01.

[39] Liu ZX, Govindarajan S, Kaplowitz N. Innate immune system plays a critical role in determining the progression and severity of acetaminophen hepatotoxicity. Gastroenterology 2004;127:1760-1774.

[40] Yiang GT, Yu YL, Lin KT, Chen JN, Chang WJ, Wei CW. Acetaminophen induces JNK/p38 signaling and activates the caspase-9-3-dependent cell death pathway in human mesenchymal stem cells. Int J Mol Med 2015;36: 485-492. doi: 10.3892/ijmm.2015.2254.

[41] Win S, Than TA, Min RW, Aghajan M, Kaplowitz N. c-Jun N-terminal kinase mediates mouse liver injury through a novel Sab (SH3BP5)-dependent pathway leading to inactivation of intramitochondrial Src. Hepatology 2016; 63:1987-2003. doi: 10.1002/hep.28486.

[42] Zhang J, Zhang S, Bi J, Gu J, Deng Y, Liu C. Astaxanthin pretreatment attenuates acetaminophen-induced liver injury in mice. Int Immunopharmacol 2017;45:26-33. doi: 10.1016/j.intimp.2017.01.028.

[43] Tan CY, Saw TY, Fong CW, Ho HK. Comparative hepatoprotective effects of tocotrienol analogs against drug-induced liver injury. Redox Biol 2015;4: 308-320. doi: 10.1016/j.redox.2015.01.013.

[44] Abdel-Azeem AS, Hegazy AM, Ibrahim KS, Farrag AR, El-Sayed EM. Hepatoprotective, antioxidant, and ameliorative effects of ginger (Zingiber officinale Roscoe) and vitamin E in acetaminophen treated rats. J Diet Suppl 2013;10:195-209. doi: 10.3109/19390211.2013.822450.

[45] Mahmoud YI, Mahmoud AA, Nassar G. Alpha-lipoic acid treatment of acetaminophen-induced rat liver damage. Biotech Histochem 2015;90:594-600. doi: 10.3109/10520295.2015.1063005.

[46] März W, Wieland H. HMG-CoA reductase inhibition: anti-inflammatory effects beyond lipid lowering? Herz 2000;25:117-125.

[47] Xu H, Li XL, Yue LT, Li H, Zhang M, Wang S, et al. Therapeutic potential of atorvastatin-modified dendritic cells in experimental autoimmune neuritis 
Mizrahi M. et al: Safe APAP and statins

by decreased Th1/Th17 cytokines and up-regulated T regulatory cells and NKR-P1(+) cells. J Neuroimmunol 2014;269:28-37. doi: 10.1016/j.jneuroim.2014.02.002

[48] Arnaud C, Burger F, Steffens S, Veillard NR, Nguyen TH, Trono D, et al. Statins reduce interleukin-6-induced $C$-reactive protein in human hepatocytes: new evidence for direct antiinflammatory effects of statins. Arterioscler Thromb Vasc Biol 2005;25:1231-1236. doi: 10.1161/01.ATV.0000163840.63685.0c.
[49] Mühl H. STAT3, a key parameter of cytokine-driven tissue protection during sterile inflammation - the case of experimental acetaminophen (paracetamol)induced liver damage. Front Immunol 2016;7:163. doi: 10.3389/fimmu.2016. 00163.

[50] Feng D, Wang $Y$, Wang $H$, Weng $H$, Kong $X$, Martin-Murphy BV, et al. Acute and chronic effects of IL-22 on acetaminophen-induced liver injury. J Immunol 2014;193:2512-2518. doi: 10.4049/jimmunol.1400588. 